\title{
PSYCHOSOCIAL STRESS AND QUALITY OF LIFE IN PATIENTS WITH TYPE 2 CARDIORENAL SYNDROME
}

\author{
Elena BIVOL ${ }^{1,2 \bowtie}$, Livi GRIB ${ }^{1}$ \\ ${ }^{1}$ Department of Internal Medicine, Division of Cardiology, State University of Medicine and Pharmacy \\ „N. Testemitanu“, Chisinau, Republic of Moldova \\ ${ }^{2}$ Clinical Hospital „Sfinta Treime“, Chisinau, Republic of Moldova \\ Received 16 Nov 2018, Accepted 26 Jan 2019 \\ hitps://doi.org/10.31688/ABMU.2019.54.1.20
}

\section{Abstract}

Introduction. Multiple studies have assessed the role of the stress, as well as of the quality of life, in patients with heart failure. However, specific data about the quality of life in patients with cardiorenal syndrome (CRS) are limited.

The objective of the study. To assess the quality of life and the level of psychosocial stress in patients with type 2 cardiorenal syndrome.

Materials and methods. We performed a case-control study for the comparative analysis of two groups of heart failure (HF) patients, with mid-range or reduced ejection fraction: 78 cardiorenal syndrome patients and 64 patients with no renal impairment.

Results. The quality of life parameters was diminished in most of the patients with HF, being more noticeable in the cardiorenal group, 23.6 vs. 28.62 points ( $\mathrm{p}<0.001$ ). HF patients with renal impairment have a more altered psychological state compared to patients without renal impairment, due to depression, anxiety and reduced vitality related to heart disorders, as well as renal dysfunction (32.67 vs. 36.06 points, $p<0.05$ ). The stress level was lower in subjects with CRS compared to those with no CRS -315.57 vs. 329.97 points $(\mathrm{p}<0.05)$. Factors

\section{Résumé}

Le stress psychosocial et la qualité de vie des patients atteints du syndrome cardio-rénal de type 2

Introduction. Plusieurs études ont évalué le rôle du stress ainsi que la qualité de vie chez les patients avec insuffisance cardiaque. Cependant, les données sur le syndrome cardio-rénal sont limitées.

L'objectif de l'étude: Évaluer la qualité de vie et le niveau de stress psychosocial dans le syndrome cardio-rénal de type 2.

Matériel et méthode: Nous avons réalisé une étude cas-témoins pour une analyse comparative de deux groupes de patients avec insuffisance cardiaque présentant une fraction d'éjection moyenne ou réduite: 78 patients atteints de syndrome cardio-rénal et 64 patients ne présentant aucune affection rénale.

Résultats: Les paramètres de la qualité de la vie ont été diminués chez la plupart des patients avec insuffisance cardiaque, plus évidemment dans le groupe avec syndrome cardio-rénal de 23,6 versus 28,62 points ( $p$ $<0,001)$. Les patients avec syndrome cardio-rénal ont un état psychologique plus altéré que ceux sans insuffisance rénale, à cause de la dépression, de l'anxiété et de la perte de vitalité liées aux troubles cardiaques ainsi 
enhancing stress resistance were more common in the CRS group.

Conclusions. CRS influences patients' status significantly, mostly by diminishing the quality of life.

Keywords: heart failure, cardiorenal syndrome, quality of life, stress.

\section{Abbreviations}

HF-Heart failure

CRS-cardiorenal syndrome

CVD-cardiovascular disease

LV- left ventricle

EF-ejection fraction

eGFR-estimated glomerular filtration rate

PF- Physical Functioning

PRF-Physical Role Functioning

BP-Bodily Pain

GHP-General Health Perceptions

VT-Vitality

SRF-Social Role Functioning

ERF-Emotional Role Functioning

MH-Mental Health

\section{INTRODUCTION}

An increasing number of studies addressed the quality of life in patients with different pathologies. The study of somatic disorders influencing patients' quality of life expresses a current problem, that is the psycho-emotional and cognitive impact of somatic diseases ${ }^{2}$.

Evaluation of patients' quality of life is usually done using validated questionnaires. The abbreviated form of Medical Outcome Study questionnaire, comprising 36 items (SF-36), was used as a health indicator in the general population. The test can also be used as a qualitative result in medical practice and research. As a general tool, SF-36 was designed to be applicable to a wide variety of conditions. SF-36 is useful for monitoring patients with single or multiple pathological conditions and for comparing their status with that of the general population ${ }^{3,4}$. SF-36 items are derived from the original MOS (Rand's Medical Outcome Study) questionnaire containing 245 items. SF-36 includes multi-item scales that measure the following eight parameters: Physical Functioning (PF), Physical Role Functioning (PRF), Bodily Pain (BP), General Health Perceptions (GHP), Vitality (VT), Social Role Functioning (SRF), Emotional Role Functioning (ERF), Mental Health $(\mathrm{MH})^{5,6}$.

Chronic diseases represent the most serious cause of mortality: $60 \%$ of global deaths are caused by chronic diseases? ${ }^{7}$. In general, quality of life in patients suffering from chronic pathologies has been que de la dysfonction rénale $(32,67$ vs 36,06 points, $p$ $<0,05)$. Le niveau de stress était plus bas chez les sujets atteints de syndrome cardio-rénal que chez ceux sans problèmes rénaux - 315,57 vs 329,97 points $(p=0,05)$. Les facteurs augmentant la résistance au stress étaient plus fréquents dans le groupe avec du syndrome cardio-rénal.

Conclusions: Le syndrome cardio-rénal influe de manière significative le statut des patients, contribuant de manière importante à la diminution de la qualité de la vie.

Mots-clés: insuffisance cardiaque, syndrome cardio-rénal, qualité de vie, stress.

and continues to be the subject of worldwide interdisciplinary research (medical, psychological, social, socio-economic, etc. $)^{8}$. Results of international studies on quality of life of oncological, cardiovascular, neurological and psychiatric patients are promoted in current medical practice, in order to improve their quality of life. The importance of this problem is given by the large number of patients with chronic illnesses, the social, community and family implications, as well as the economic problems raised by providing care to these patients.

Due to limited data on the impact of cardio-renal syndrome (CRS) upon quality of life ${ }^{7}$, we focused our attention on the initial pathology of the respective syndrome - heart failure? . Heart failure (HF) has a negative impact on patients' prognosis and quality of life ${ }^{10}$.

According to the literature data, quality of life assessment in HF has become a relevant tool in defining disease prognosis and therapeutic intervention effectiveness $^{5,10}$. Over the last decade, several studies on HF patients' quality of life have been conducted, recognizing the importance of assessing the disease, not only biologically, but also psycho-socially. A literature review identifies over 150 tools for assessing quality of life ${ }^{5,11}$.

A study on the quality of life in patients with chronic ischemic heart failure, with or without left ventricular systolic dysfunction, by Vatamanu et al, found that the signs of psychiatric disturbances and pain, as well as the total physical component, were 
more favorable in patients without left ventricle (LV) systolic dysfunction ${ }^{12}$.

A study by Lahoud et al, on a group of 7056 patients, during 1996-2011 period, detected that a low SF-36 score for the physical component was associated with a 6 -fold increase of 8 -year mortality $[95 \%$ confidence interval], $\mathrm{p}<0.001$. In a one-dimensional analysis, SF-36 physical score had a c-index of 0.75 , being superior to all biomarkers ${ }^{13}$.

In a study by Huber et al (2016), on a group of 5508 patients with angina, myocardial infarction and ischemic heart failure, the diagnosis had a significant influence on physical health perception. The highest SF-36 physical component score was reported in patients with myocardial infarction, while the lowest score in patients with heart failure. Lower physical health values were obtained in women, elderly patients (especially $\geq 70$ years) and patients who reported an elevated ERF score ${ }^{14}$.

Perlman et al performed a multicenter observational study on 634 patients with chronic kidney disease (CKD). SF-36 scores were higher in the cohort of patients with CKD compared to hemodialysis patients, but lower than the control group. Glomerular filtration rate was not significantly associated with quality of life. The level of hemoglobin correlated with both SF-36 physical and mental components ${ }^{15}$.

\section{Impact of psychosocial stress on patients with cardiac and renal diseases}

Cardiovascular disorders continue to be the most important cause of death and disability all over the world. Unlike somatic risk factors, the study of psychosocial factors in patients with cardiovascular disease was somewhat neglected ${ }^{15}$. Convincing data obtained by Dimsdale (2008) linked depression, social isolation, stress, anger, and anxiety, as well as a number of other psychosocial factors, to both coronary heart disease onset and evolution ${ }^{16}$. Several studies assessed the relationship between stress, stressful events, or other psychosocial factors and acute myocardial infarction onset. A study by Nahshoni et al (2005) concluded that mental stress was significantly correlated to myocardial events (both fatal and non-fatal), as well as heart failure worsening, regardless of age and/or ejection fraction ${ }^{17}$.

A prospective study by Rod et al (Denmark), regarding psychosocial factors role in heart failure development, aimed to address the effect of psychosocial factors on heart failure hospitalization rate. Of the 8,670 enrolled participants (1991-1993), 20\% had a certain degree of vital exhaustion which has been linked to an increased risk of heart failure repeated hospitalization. Major life events, social conditions, and sleeping pills during hospitalization did not influence the hospitalization rate ${ }^{18}$.

\section{THE OBJECTIVE OF THE STUDY}

To assess quality of life and level of psychosocial stress in type 2 cardiorenal syndrome.

\section{Materials AND MEthods}

We performed a case-control study for comparative analysis of two groups of heart failure patients, with mid-range or reduced ejection fraction (EF). We enrolled eligible patients who were hospitalized in the Cardiology Units of the Municipal Clinical Hospital "Sfanta Treime“, Chisinau, Republic of Moldova, during 2016-2018 period. The experimental protocol No. $11(09.12 .2015)$ was approved by the Ethics Committee of the State University of Medicine and Pharmacy „Nicolae Testemitanu“, Chisinau.

\section{Inclusion criteria:}

- Chronic heart failure (CHF) diagnosis (as defined by the 2016 Europea Society of Cardiology Guidelines for the diagnosis and treatment of acute and chronic heart failure $)^{19}$.

- Left ventricle ejection fraction (LVEF) $\leq 49 \%$.

- No renal impairment (chronic kidney disease CKD) or documented onset of heart failure prior to renal impairment onset.

- Patient's informed consent.

- Age over 18 years.

\section{Exclusion criteria:}

- Presence of primary kidney disease (congenital kidney disease or kidney disorders prior to cardiac disease).

- Inflammatory, traumatic kidney disease (that cannot be explained by chronic heart failure).

- Malignant tumors.

- Steroid treatment.

- Patients with acute cardiac/cerebrovascular events.

- Psychiatric disorders or dementia.

- Patient's refusal.

Eighty-three patients were included in the cardiorenal syndrome group, while control group was comprised of 66 patients with HF with no CRS. Diagnosis was established through calculation of estimated glomerular filtration rate (GFR) by means of CKD-EPI equation, based on Cystatin C and Creatinine serum levels $\left(\mathrm{ml} / \mathrm{min} / 1.73 \mathrm{~m}^{2}\right)=$ GFRcyscr $=135 \times \min (\mathrm{Scr} / \kappa, 1) \alpha \times \max (\mathrm{Scr} / \kappa$, 1) $-0.601 \times \min ($ Scys $/ 0.8,1)-0.375 \times \max ($ Scys $/ 0.8$, 1) $-0.711 \times 0.995$ Age 0.969 [if women] $\times 1.08$ [if 
black], where Scr stands for serum creatinine, $\kappa$ is 0.7 for women and 0.9 for men, $\alpha-0.248$ for women and -0.207 for men, min indicates the minimum value for $\mathrm{Scr} / \mathrm{\kappa}$ or 1 , max indicates the maximum value for $\mathrm{Scr} / \mathrm{\kappa}$ or 1 , and Scys stands for serum Cystatin C. Type 2 cardiorenal syndrome was established in patients with eGFR $<60 \mathrm{ml} / \mathrm{min} / 1.73 \mathrm{~m}^{2} .{ }^{20}$

- The Social Readjustment Rating Scale (SRRS) highlights stress and stressful events by recording difficult or even tragic life events. Thomas Holmes and Richard Rahe (1967) developed a questionnaire-based process that includes a list of 43 such events, each event being assigned a fixed value that can be used as a stress indicator ${ }^{21}$.

- The Perceived Stress Scale (PSS-10) developed by Cohen, Kamarck and Mermelstein (1983, apud Taylor, 1998) contains 10 statements about whether one feels stressed or unprepared for one's requirements. This results in a sum total that indicates the degree of stress ${ }^{22}$.

- General Well-Being Index.

- Quality of life was assessed using the short SF-36 questionnaire. SF-36 items derive from the original MOS (Rand's Medical Outcome Study) questionnaire containing 245 items. SF-36 includes multi-item scales that measure the following eight parameters: Physical Functioning (PF), Physical Role Functioning (PRF), Bodily Pain (BP), General Health Perceptions (GHP), Vitality (VT), Social Role Functioning (SRF), Emotional Role Functioning (ERF), Mental Health (MH).

- Questionnaire highlighting factors diminishing and enhancing stress resistance ${ }^{23}$.

Statistical analysis was performed using SPSS 20.0 and Microsoft Office Excel 2010.

\section{Results AND DISCUSSION}

The data provided in Table 1 denote a male predominance in the study, both in the CRS group (51.8\%) and in the one with no CRS (69.7\%), (Pearson's correlation coefficient $\mathrm{r}=0.18$, statistically significant data, $\mathrm{p}<0,05)$. Both study groups had an urban residence predominance, with $72.3 \%$ and $74.2 \%$, respectively.

Next, we analyzed the gender distribution. While examining patients from Swedish HF registry, during 2000-2013 period, Lofman noticed a low prevalence among women: $27 \%$ for eGFR $\geq 90 ; 35 \%$ for eGFR in the $60-89$ range; $44 \%$ for eGFR $30-59$; $42 \%$ for eGFR < 15. The group with an eGFR between 15 and 29 had a symmetric distribution $-50 \%{ }^{24}$. The CHART study investigated a population of $920 \mathrm{HF}$ subjects with $\mathrm{LVEF} \leq 50 \%$ and reported an increased prevalence of CRS in men: $69.4 \%$ for eGFR $\geq 60$;
$60.5 \%$ for eGFR 30-59; and $53.1 \%$ for eGFR $<30^{25}$. Kumar noticed an increased prevalence of all types of CRS among men, as well ${ }^{26}$.

In our study, we found CRS to be present in $48.2 \%$ women and $51.8 \%$ men, the data being statistically significant $(\mathrm{p}<0.001)$. Next, we analysed CRS frequency in both genders according to CRS severity. Thus, among patients with CRS with an eGFR $>60$, men were 46 (69.7\%), in those with CRS with an eGFR between 30-60, men were 41 (59.42\%) and among CRS patients with eGFR < 30, women predominated 12 (85.7\%) (Pearson coefficient $\mathrm{r}=0.18$; $\mathrm{p}<0.05$ )

The impact of CRS on quality of life appreciated by SF-36 questionnaire

The impact of locomotor system impairment on quality of life in patients with cardiorenal syndrome was assessed using the SF-36 questionnaire, a general tool designed to be applicable to a wide variety of conditions. Upon applying this tool, the obtained standardized results were scored on a scale of 0 to 100 , with 0 being the worst-case scenario and 100 the best one. Each question scores are encoded in multiple steps, depending on interrogation items number.

Both heart failure and chronic kidney disease lead to a decrease in patients' quality of life. Our goal was to assess quality of life in patients diagnosed with both pathologies and analyse whether their synergism decreases or maintains quality of life at the same level compared to patients diagnosed with heart failure without renal impairment (Table 2).

We determined that SF-36 components had lower values in the study group than in the control group, the latter mean value being below 50 points, which means that both CRS patients and HF patients without renal impairment had a significant physical and mental burden. Except for mental health component, quality of life indices had statistically significant differences between groups.

As a result, SF-36 scores for PF, PRF, BP, GHP, VT, SRF, ERF, and MH parameters in patients with $\mathrm{HF}$ and associated renal disease were: $9.04 \pm 1.32$; $17.31+3.45 ; 21.39 \pm 1.99 ; 15.71 \pm 2.04 ; 15.06 \pm 1.56$; $25.49 \pm 2.84 ; 33.78 \pm 4.61$ and $51.03 \pm 1.54$. The mean general health score was 23.6. In the group without CRS, scores for the same parameters were: $15,39 \pm$ 2,$16 ; 23.91 \pm 3.88 ; 26.51 \pm 2.54 ; 20.70 \pm 2.29 ; 18.59$ $\pm 2.23 ; 31.49 \pm 2.96 ; 39.06 \pm 4.73$ and $53.31 \pm 1.95$ respectively. The mean general health score was 28.62 .

It is evident that the quality of life in the main group patients with HF and kidney disease is much lower compared to control group patients, with HF but no kidney disease. Comparative analysis results of the mean PF value in patients with CRS and without 
it were the following: $9.04 \pm 1.32$ vs. $15.39 \pm 2.16$ ( $\mathrm{p}<$ 0.001 ). The gap between scales was 6.35 for PF and 6.6 for PRF, indicating that in patients with renal impairment on top of HF, the negative impact on quality of life is explained by physical activity limitations, work or other daily activities capacity reduction resulting from physical health.

The performed physical activity mean value, according to the Physical Functioning Scale, was significantly dependent upon cerebral natriuretic peptide level and LVEF, but not influenced by patient's age. The Physical Role Functioning (PRF) Scale, including items that underline whether there is a reduction in working time, limitations in the nature of work, or whether there is difficulty in performing daily activities, found out that some patients reduced the number of activities, as well as limited the nature of their work. A statistically significant difference $(p<0.01)$ in patients with CRS $(17.31 \pm 3.45)$ and in those without CRS (23.91 \pm 3.88$)$ was found upon evaluation of the previous scale. We found a moderate, inverse, statistically significant correlation with NT pro-BNP level ( $\mathrm{r}$ $=0.35, p<0.001)$, HF degree $(r=0.27 ; p<0.05)$ and age $(r=0.25, p<0.05)$; a moderate, direct correlation with patient's mobility level $(r=0.49, p<0.001)$, vitality level $(\mathrm{r}=0.57, \mathrm{p}<0.0001)$ and mild correlation with eGFR $(r=0.24, p<0.05)$.

Statistical analysis of bodily pain severity (BP) scale data showed a statistically significant difference ( $p<0.01)$ between study groups - patients with renal impairment $(21.39 \pm 1.99)$ and HF patients without renal impairment (26.51 \pm 2.54$)$. As a result of Pearson's correlation analysis, we noted a moderate, inverse, statistically significant correlation with NT pro-BNP level $(r=0.46 ; p<0.001)$ and HF degree $(r$ $=0.4 ; \mathrm{p}<0.001)$ and a mild, direct correlation with patient's mobility level $(r=0.36, p<0.001)$ and eGFR $(\mathrm{r}=0.29, \mathrm{p}<0.05)$

The general health scale (GH), i.e. assessment of one's own health, in study group patients was $70 \%$, considered to be mediocre. No patient perceived general health as „very good“. The perception of their own health was an equally important parameter included in this questionnaire $(\mathrm{GH})$, reflecting patients' quality of life: in patients with CRS, score values were $21.39 \pm 1.99$, compared to patients without CRS $-26.51 \pm 2.54$, the difference being statistically significant $(\mathrm{p}<0.01)$. In the study group, general health status correlated with NT proBNP levels $(r=$ $0.43, p<0.001$ ), a statistically significant inverse correlation. We found a mild correlation with age $(\mathrm{r}=0.25$, $\mathrm{p}<0.05)$, mobility degree $(\mathrm{r}=0.32 ; \mathrm{p}<0.01)$, physical role functioning $(r=0.27, p<0.05)$, and social role functioning $(r=0.56, p<0,001)$. When it came to pain intensity $(\mathrm{r}=0.27, \mathrm{p}<0.05)$ and stress intensity $(-0.229$
0.0478), we found inverse, mild, statistically significant correlations. The degree of renal impairment in the study group did not significantly affect general health status and eGFR $(r=0.13, p<0.26)$.

The vitality scale (VT) showed that vitality and energy were seldom or never felt by patients with heart failure, with or without SCR, with fatigue or exhaustion predominance. The comparison of energy level between the main group $(15.06 \pm 1.56)$ and the control group (18.59 \pm 2.23$)$ was statistically significant $(\mathrm{p}<0.001)$. In the study group, vitality correlated inversely, statistically significant with NT proBNP levels $(r=0.37, p<0.001)$. Statistically significant mild correlations were observed with $\operatorname{LVEF}(\mathrm{r}=0.29, \mathrm{p}$ $<0.01)$, patient mobility degree $(\mathrm{r}=0.40, \mathrm{p}<0.01)$, physical functioning $(r=0.34, p<0.01)$, social role functioning $(r=0.65, p<0.001)$, and emotional role functioning $(\mathrm{r}=0.57, \mathrm{p}<0.001)$.

The social role functioning (SRF) scale demonstrated that physical health status or emotional problems affected everyday social activities with family, friends, neighbors, and during the last 4 weeks there have been times when physical or emotional health has affected social activities (such as visiting friends, relatives). Scale values for the extent and frequency of social activity obstacles due to physical and emotional problems showed statistically significant difference in patients with HF with or without renal impairment (25.49 \pm 2.84 and $31.49 \pm 2.96, \mathrm{p}>0.05)$. Correlation analysis, according to Pearson method, revealed a high statistically significant correlation with vitality degree $(r=0.76, p<0.001)$, pain level $(r$ $=0.77, \mathrm{p}<0.001)<0.001)$, physical activity $(\mathrm{r}=0.63, \mathrm{p}$ $<0,001)$ and emotional state $(r=0.66, p<0.001)$. A mild, inverse correlation $(\mathrm{r}=0.33, \mathrm{p}<0.01)$ with HF degree was also identified.

Emotional role functioning (ERF) in patients with HF with renal impairment had a scale value of $33.78 \pm 4.61$, while in patients with HF with no eGFR reduction, the value was $39.06 \pm 4.73$, the difference being statistically significant ( $\mathrm{p}<0.001$ ).

Mental health $(\mathrm{MH})$ scale had „sometimes' as the main response, patients feeling depressed for a long time, in particular patients from the first and second study groups. Feelings of nervousness and depression in patients with or without CRS did not reveal a statistically significant difference $(51.03 \pm 1.54$ vs. $53.31 \pm 1.95, \mathrm{p}>0.05)$. The feeling of nervousness and sadness in the main group patients with CRS moderately correlated with exhaustion $(r=0.57, p$ $<0.001)$, general health $(r=0.55, p<0.001)$ and social role functioning $(r=0.4, p<0.001)$. Mild, statistically significant, inverse correlations were observed for financial state $(r=0.24, p<0.05)$ and stress intensity $(r$ $=0.37, \mathrm{p}<0.001)$. 
In this way, our data show that, in HF patients with renal impairment, routine physical activities performed over the course of the day have a more direct impact on working capacity compared to patients without renal impairment, by reducing the time dedicated to work or other activities due to fulfillment difficulties. The study group had also a more pronounced impairment of vitality state and emotional state manifested through depression, restlessness and unhappiness.

Thus, CRS influences the patient's psycho-emotional and physical status significantly contributing to motor physical disturbances.

\section{General Well-Being Index (GWB)}

Chronic presence of cardiovascular and renal symptoms, such as progressive dyspnea, edema, chronic pain, impair patients' quality of life under several aspects, as highlighted by SF-36 questionnaire. Indeed, they create an impediment not only in performing routine daily activities and having an active lifestyle, but also, being permanent, they have a negative impact on patients' psychological state. This fact led us to assess this quality of life indicator as well, determined by disease impact on the general well-being psychological status, that was evaluated by means of the General Well Being (GWB) generic questionnaire. It involved evaluation of six impact areas: anxiety, depression, general health status, well-being, self-control and vitality. The results were interpreted based on a total score of 0 to 110 points, which was separately calculated for the two groups of patients.

The mean overall score for the CRS group was 32.67 points $(95 \%$ CI $29.43-35.9)$ vs. 36.06 points (95\% CI 32.09 - 40.03) for the control group, both results standing for severe suffering state. Mean scores difference was statistically significant $(\mathrm{p} \leq 0.05)$ for the study group, demonstrating a more altered quality of life among patients with CRS. When dividing the total GWB questionnaire score into three levels, depending on psychological suffering severity, we obtained the following distribution: severe suffering $(0$ to 60 points) was present in 73 patients (93.6\%) with CRS and 58 (87.88\%) without CRS; moderate suffering (61-72 points) present in 5 patients $(6.4 \%)$ in the first group and $6(9.1 \%)$ in the control group; no study patient had good psychological status (73-110 points). Thus, we found that patients with HF and renal impairment have a more altered psychological state compared to patients without renal impairment, due to depression, anxiety, and reduced vitality level associated with both cardiac and renal dysfunctions.

\section{Social Readjustment Rating Scale (SRRS)}

Important life events, both positive (such as marriage) and negative (such as death of a close friend) cause stress. Interpretation of stress levels is difficult because of great differences in each person's ability to cope with it, as well as their stress-specific reactions. Thomas Holmes and Richard Rahe developed a questionnaire by listing common stressful events and arbitrarily assigning values of „life changing units“.

The mean general score for the CRS group was $315.57 \pm 13.68$ points $(\mathrm{p}=0.72)$ vs. $329.97 \pm 17.07$ points $(\mathrm{p}<0.05)$ in control group patients, both standing for high stress levels. Upon dividing the SRRS questionnaire total score in three levels depending on stress severity, we obtained the following distribution: light stress (0 to 149 points) with low probability (30\%) (128) of developing stress-induced pathologies was present in 7 CRS patients (9.1\%) and in 4 (6.25\%) of those without CRS; moderate stress (150-299 points) with a $50 \%$ risk of developing stress-induced pathologies was present in 29 patients (37.66\%) from the main group and 22 control group patients (34.38\%); intense stress (> 300 points) with a $80 \%$ risk of developing stress-induced pathologies was present in 41 patients $(53.25 \%)$ from the main group and 38 (48.44\%) from the control group. We have found a moderate, inverse correlation between stress levels estimated by SRRS and pain level $(r=0.49, p<0.001)$, vitality $(r=0.45, p$ $<0.001)$, physical functioning $(r=0.41, p<0.001)$ and emotional state $(r=0.43, p<0.001)$. In addition, mild, inverse correlations were noted for age $(r=0.32, p$ $<0.01)$, general health $(r=0.38, p<0.001)$, SF-36 social role functioning $(r=0.35, p<0.001)$.

\section{Perceived Stress Scale (PSS-10)}

The scale contains 10 statements about the state of stress or unpreparedness for certain requirements, which indicate the degree of experienced stress.

The mean general score for the CRS group was $23.82 \pm 0.63$ points ( $\mathrm{p}<0.001$ ) vs. $22.59 \pm 0.57$ points ( $\mathrm{p}$ $=0.38$ ) in control group patients without renal impairment, both results standing for moderate stress levels. When stratifying PSS-10 questionnaire total score according to stress levels, we noticed that severe stress ( 227 points) was present in 22 CRS group patients (28.57\%) and 10 control group patients (15.63\%). Even if, compared to SRRS questionnaire, stress level was lower, both scales showed a predominance of cases with increased stress levels among patients with CRS. We found a moderate, inverse correlation between stress level assessed by PPS-10 and general well-being index (GWB: $r=0.65, p<0.001$ ), pain level $(r=0.45, p<0.001)$, vitality assessed by SF questionnaire $(\mathrm{r}=0.44, \mathrm{p}<0.001)$, social role functioning $(\mathrm{r}=$ $0.49, \mathrm{p}<0.001)$, general health $(\mathrm{r}=0.44, \mathrm{p}<0.001)$ and 
emotional role functioning $(\mathrm{r}=0.46, \mathrm{p}<0.001)$. In addition, we found a direct correlation for the stress level assessed by SRRS questionnaire $(r=0,45, p<0.001)$ and social role functioning $(\mathrm{r}=0.35, \mathrm{p}<0.001)$.

\section{Questionnaire highlighting factors diminishing and enhancing stress resistance (108)}

The Social Readjustment Rating Scale is a simple and relatively objective tool for assessing stress by listing important stressful events. Several psychologists have questioned this assessment method, stating that the rarity of these events cannot explain recurrent every day stress that people feel. This stress is caused by minor tensions with relatives and colleagues, such as hiring, litigation with a husband or colleague, lack of a train, etc. They think that such events could help counteract the negative effect of daily negative situations on an individual's stress level.

The mean general score highlighting factors diminishing stress resistance was $14.92 \pm 0.35$ points ( $p$ $<0.05$ ) for the CRS group vs. $15.51 \pm 0.43$ points ( $p>$ 0.05 ) for the control group. The mean general score highlighting factors enhancing stress resistance was $18.53 \pm 0.43$ points $(p>0.05)$ for the CRS group and $16.97 \pm 0.44$ points ( $p>0.05$ ) for the control group. The results confirm SF-36, GWB and SRRS questionnaire data, the recorded stress level being lower in CRS subjects compared to those without CRS. It should be pointed out that in the CRS group, stress resistance enhancing factors were more frequent, but with no significant impact on general health $(r=0.22$, $p>0.05)$. A significant correlation with perceived stress was noted $(r=0.43, p<0.001)$.

\section{Conclusions}

In most patients with HF, quality of life indices was diminished, being more noticeable in the CRS group. Patients with HF and renal impairment had a more altered psychological state compared to patients without renal impairment, due to depression, anxiety, and reduced vitality associated with both cardiac and renal dysfunctions. The assessed stress level was lower in subjects with CRS compared to those without it. Stress resistance enhancing factors were more frequent in the CRS group. CRS influences patient's status significantly, especially contributing to motor physical disturbances.

\section{Compliance with Ethics Requirements:}

„The authors declare no conflict of interest regarding this article"

"The authors declare that all the procedures and experiments of this study respect the ethical standards in the
Helsinki Declaration of 1975, as revised in 2008(5), as well as the national law. Informed consent was obtained from all the patients included in the study"

"No funding for this study"

\section{References}

1. Karner-Hutuleac A. Aspecte etice ale cercetarii calitatii vietii pacientilor cronici. Revista Romana de Bioetica 2013;1(11):79-87.

2. Sinescu I, Manu MA, Harza M, et al. Studiul calitatii vietii folosind „SF-36 HEALTH SURVEY TEST" la pacientii transplantati, pacientii hemodializati, pacientii uremici cu urolitiaza maligna. Revista Romana de Urologie 2008; 3:1-6.

3. Cella D, Nowinski CJ. Measuring quality of life în chronic illness: the functional assessment of chronic illness therapy measurement system. Arch Phys Med Rehabil 2002; 83:S10-17.

4. Gill TM, Feinstein AR. A critical appraisal of the quality of quality of life measures. JAMA 1994; 272:619-26.

5. Anker SD, Agewall S, Borggrefe M, et al. The importance of patient-reported outcomes: A call for their comprehensive integration în cardiovascular clinical trials. European Heart Journal 2014;35(30):2001-2009.

6. Tintiuc D, Grejdian T, Margine L, et al. Factorii determinanti - ce contribuie la o speranta de viata longeviva printr-un mod de viata sanatos. Buletinul Academiei de Stiinte a Moldovei - Stiinte medicale 2014;4(45):212-216.

7. Efremova EV, Shutov AM. Clinical characteristics, quality of life and prognosis of patients with chronic cardiorenal syndrome. (Published in Russian). Nephrology 2015; 2(19):63-67.

8. Nechita AM, Radulescu D, Peride I, et al. Determining factors of diuresis in chronic kidney disease patients initiating hemodialysis. Journal of Medicine and Life 2015;8(3):371-377.

9. Heo S, Lennie TA, Okoli C, Moser D. Quality of life in patients with heart failure: ask the patients. Heart Lung 2009;38(2):100-108.

10. Alonso J, Ferrer M, Gandek B, et al. Health-related quality of life associated with chronic conditions in eight countries: results from the International Quality of Life Assessment (IQOLA) Project. Quality of Life Research 2004;13(2):283-98.

11. Cruz DN, Bagshaw SM. Heart-kidney interaction: epidemiology of cardiorenal syndromes. International Journal of Nephrology 2011; 2011:7.

12. Vataman E, Lisii D, Filimon S, Priscu O. Studiul calitatii vietii la pacientii cu insuficienta cardiaca cronica de origine ischemica cu sau fara disfunctie sistolica a ventriculului stang. Curierul Medical 2012; 3(327):370-373.

13. Lahoud R, Chongthammakun V, Wu Y, Hawwa N, Brennan DM, Cho L. Comparing SF-36 scores versus biomarkers to predict mortality in primary cardiac prevention patients. European Journal of Internal Medicine 2017;46:47-55.

14. Huber A, Oldridge N, Hofer S. International SF-36 reference values in patients with ischemic heart disease. Quality of Life Research 2016;25:2787-2798.

15. Perlman R, Finkelstein F, Liu L, Roys E, Kiser M. Quality of life in Chronic Kidney Disease (CKD): A cross-sectional analysis in the Renal Research Institute-CKD study. American Journal of Kidney Disease 2005;45(4):658-666.

16. Wei J, Rooks C, Ramadan R et al. Meta-analysis of mental stress-induced myocardial ischemia and subsequent cardiac events in patients with coronary artery disease. American Journal of Cardiology 2014;114(2):187-192. 
17. Dimsdale E. Psychological stress and cardiovascular disease. Journal of the American College of Cardiology 2008;51(13):12371246.

18. Rod NH, Andersen I, Prescot E. Psychosocial risk factors and heart failure hospitalization: a prospective cohort study. Am J Epidemiol 2011;174(6):672-680.

19. Ponikowski P, Voors AA, Anker SD, et al. ESC Guidelines for the diagnosis and treatment of acute and chronic heart failure. The Task Force for the diagnosis and treatment of acute and chronic heart failure of the European Society of Cardiology (ESC). European Heart Journal 2016; 37(27): 2129-2200.

20. Ronco C, McCullough P, Anker S, et al. Cardio-renal syndromes: report from the consensus conference of the Acute Dialysis Quality Initiative. European Heart Journal 2010; 31(6): 703-711.

21. Holmes TH, Rahe RH. The social readjustment rating scale. Journal of Psychosomatic research 1967;11(2):213-218.
22. Cohen S, Kamarck T, Mermelstein R. A global measure of perceived stress. Journal of Health and Social Behavior 1983;24:385-396

23. DeLongis A, Folkman S, Lazarus R. The impact of daily stress on health and mood: Psychological and social resources as mediators. Journal of Personality and Social Psychology 1988; 54:486-495.

24. Löfman I, Szummer K, Hagerman I, Dahlström U, Lund LH, Jernberg T. Prevalence and prognostic impact of kidney disease on heart failure patients. Open Heart 2016;3(1):1-9.

25. Shiba N, Matsuki M, Takahashi J, Tada T. Prognostic importance of chronic kidney disease in Japanese patients with chronic heart failure. Circulation Journal 2008;72:173 -178.

26. Kumar S. Prevalence of cardiorenal syndrome in patients admitted to a tertiary hospital. International Journal of Pharma and Bio Sciences 2016; 7(4):517 - 521. 